\title{
Hartmut Krafft
}

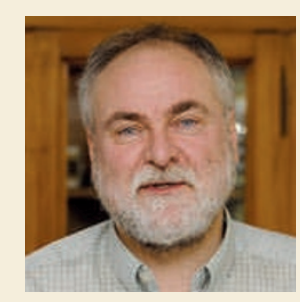

Head of Clinical Trials Unit, Paul-Ehrlich-Institut, Langen, Germany. Prior to becoming Head of the Clinical Trials Unit, Hartmut Krafft was involved in the assessment of antibodies, batch-release testing and regulatory affairs at the Institute, which is the Federal Institute for Vaccines and Biomedicines in Germany. Alongside his current role, he is a member of several working parties involved in clinical trials and is presently the Co-chair of the European Clinical Trials Facilitation Group.

Why and when was the Voluntary

Harmonisation Procedure (VHP) for the assessment of multinational clinical trials established?

When the clinical trials directive came into force in the European Union (EU) in 2004, the Heads of Medicines Agencies established the Clinical Trials Facilitation Group (CTFG) to support the authorization of clinical trials across the member states. One important request to the European Medicines Agency was to issue alerts to national competent authorities (NCAs) - the agencies that assess clinical trial applications in each member state; for example, the Medical Products Agency in Sweden and the Paul-Ehrlich-Institut (PEI) in Germany - from the clinical trials database EudraCT [which was established through the directive and is not currently publicly accessible] whenever there was a negative decision or withdrawal about a clinical trial in any of the member states. Through the alerting system we found that there were divergent decisions being made about the same clinical trials in different member states.

When the CTFG started to explore this issue we were immediately faced with the problem that we could not discuss the clinical trial applications with the different NCAs at the same time. This was because the applications were submitted to the various NCAs at different times.

The only solution was to offer sponsors a voluntary mechanism by which they could submit a clinical trial application to the CTFG and, at the same time, name all the countries in which they would like to perform the clinical trials. Then the different NCAs would assess the application together. This formed the basis of the VHP, for which the first discussions took place in 2007 and the CTFG started offering the procedure in February 2009. The PEI acts as coordinator of the process.
Now, once a sponsor has received a positive decision about a clinical trial through the VHP, they still have to apply to each member state to run the clinical trial. However, we thought that this procedure would provide a level of harmonization to prevent the applicants receiving different questions from the member states, and also to avoid having varying reasons for non-acceptance of a clinical trial application.

\section{Who can apply for a VHP and how do they apply?}

Any clinical trial sponsor, commercial or non-commercial, can apply for a VHP if they are planning to submit a clinical trial application to at least three EU member states. When we first offered the procedure, we stated that the multinational clinical trial had to be either a first-in-human clinical trial or a trial for a critical product (that is, an investigational medicinal product with a novel mode of action, a novel manufacturing process or novel administration). However, the only restriction we now have is that the application has to be destined for at least three EU countries.

We decided not to charge application fees for participating in the VHP because we were concerned that this would discourage sponsors to use the process. As the assessment of the trial will be done once, we came to the conclusion that a sponsor should be charged by the NCAs only.

What have been the main outcomes so far? The first outcome is that the timelines for applying to do a multinational clinical trial are more rapid through the VHP than applying to each country individually. We averaged 52 days to complete the VHP for standard clinical trial applications and rarely used more than 60 days. This was true even for a trial that involved 18 countries for which we completed the VHP decision process within 50 days.

The second outcome is that once the scientific discussion about a clinical trial protocol has taken place through the VHP, that discussion will not take place again when the sponsor submits the application to the NCA. So the sponsor will not receive unexpected questions from the NCA once the clinical trial documents have been agreed through the VHP. This also enabled us to cut the assessment time from 60 to 10 days, at the national level, for any trials that have received a positive decision through the VHP.

\section{Do you think the VHP could help address challenges with Europe's clinical trial directive, and, if so, how?}

Yes I do, because the VHP offers what many stakeholders have requested: a 'one stop shop' to gain a positive decision for a multinational clinical trial. The current legal framework does still require the sponsors to apply to each NCA for national authorization of their clinical trials. However, we think that we have used the current framework in a pragmatic way to solve many of the problems that sponsors have with the clinical trials directive. We are now confident that we offer a highly attractive alternative to the system of separately applying to each member state.

We are going to try to improve the process to more efficiently use our resources. But, as this is a voluntary procedure for both the sponsors and the member states, it can be improved very quickly by the agreement of all. We do not have to change laws to change the way we conduct the VHP.

One of the major issues of the clinical trials directive that the VHP does not solve is the fact that as well as applying to the NCA in each member state to gain approval of a clinical trial, sponsors also have to apply to the respective ethics committees. It may be a good idea to submit applications to the VHP and the ethics committees at the same time. But we are only just starting to have discussions with some of the relevant organizations in the member states to determine whether ethics committees would be involved in the VHP assessments as well. Interview by Bethan Hughes 